\title{
A multicomponent dark matter scenario and the experimental evidence supporting it
}

\author{
Dylan Blend, Reagan Thornberry, Alejandro Arroyo, Gabriel Frohaug, Caden \\ LaFontaine, and Roland E. Allen* \\ Department of Physics and Astronomy, Texas A\&M University, College Station, Texas, USA \\ E-mail: w208127718@tamu.edu, rethornberryagmail.com, \\ alejandro777@tamu.edu, caden.lafontaine@tamu.edu, \\ gabriel.frohaugeicloud.com, allen@tamu.edu
}

\begin{abstract}
We review a dark matter scenario with a number of favorable aspects: (1) all of the well-known successes of supersymmetry are preserved, (2) the parameters can satisfy naturalness, (3) the addition of an extended Higgs sector implies a doubly rich plethora of new particles and new physics to be discovered in the near or foreseeable future, (4) the mass of the dominant dark matter WIMP is $\leq 125 \mathrm{GeV} / \mathrm{c}^{2}$, (5) the gauge couplings of this particle are precisely defined, and (6) naturalness implies that its Higgs-mediated couplings are also comparable to those of a natural neutralino. Recent (and earlier) analyses of the data from Planck, Fermi-LAT, AMS-02, and other experiments indicate that (i) the positron excess at $\sim 800 \mathrm{GeV}$ or above is not evidence of highmass dark matter particles (which would have disconfirmed the present theory with a rigorous upper limit of $125 \mathrm{GeV}$ ), (ii) the Galactic center excess of gamma rays observed by Fermi is evidence for dark matter particles with a mass below or near $100 \mathrm{GeV}$, (iii) the gamma-ray excess from Omega Centauri is similar evidence of annihilation of such relatively low-mass particles, and (iv) the antiproton excess observed by AMS is again evidence of $\lesssim 100 \mathrm{GeV}$ dark matter particles. The present scenario, with two stable spin 1/2 WIMPs (a high-mass neutralino and a more abundant "Higgson" with a mass of $\leq 125 \mathrm{GeV} / \mathrm{c}^{2}$ ) is consistent with these results (as well as all others which have been verified), and it also suggests that detection should be near in a variety of experiments for direct, indirect, and collider detection.
\end{abstract}

European Physical Society Conference on High Energy Physics - EPS-HEP2019 -

10-17 July, 2019

Ghent, Belgium

${ }^{*}$ Speaker. 
This is a review of a recently proposed scenario with two coexisting stable dark matter WIMPs - a neutralino [1, 2, 3] and the particle that we have called a Higgson $[4,5,6,7]$ and represented by $H^{0}$, to distinguish this variety of particle - generically labeled $H$ - from Higgs bosons $h$ and Higgsinos $\widetilde{h}$. (There are both neutral and charged particles in each of these three varieties.) Let us begin with four central theorems:

1. Both the lightest Higgson and the lightest neutralino are stable spin 1/2 WIMPs [7].

2. The lightest Higgson has a mass of $\leq 125 \mathrm{GeV} / \mathrm{c}^{2}[5,7]$.

The last two theorems, given below, require an introduction: In the extended Higgs description of Refs. $[4,5,6,7]$, Higgs fields are interpreted as the complex scalar amplitudes $\phi$ of multicomponent fields $\Phi=\phi \chi$, where $\chi$ is a constant spinor. Each $\Phi^{R}$ can be represented by a sum over the complete orthonormal set of Higgson mass eigenstates $H^{i}$, now regarded as basis functions: $\Phi^{R}=\sum_{i} \bar{c}_{R i} H^{i}$.

3. It follows that [7] the coefficient $b_{R}$ of the quartic self-coupling of this Higgs field $\phi^{R}$ is an average over the couplings $b_{R i}$ of the Higgsons $H^{i}$ to $\phi^{R}$ :

$$
b_{R}=\left\langle b_{R i}\right\rangle=\frac{\sum_{i} b_{R i}\left|\bar{c}_{R i}\right|^{2}}{\sum_{i}\left|\bar{c}_{R i}\right|^{2}} .
$$

4. For a Higgs field $\phi^{R}$ that undergoes condensation, with a vacuum expectation value $v^{R}$, there is consequently [7] a first-order coupling of a Higgson $H^{i}$ to a Higgs boson $h^{R}$ of $\phi^{R}=v^{R}+h^{R}$ :

$$
-\overline{\mathscr{L}}_{R i}=\lambda_{R i} h^{R} H^{i \dagger} H^{i}
$$

with

$$
\lambda_{R i}=2 b_{R i} v^{R}
$$

Theorems 3 and 4 suggest that [7] for the Higgson field $H^{i}$ of lowest mass (which is thus a dark matter WIMP)

$$
\lambda_{R i} \sim 2 b_{R} v^{R} \approx 2(0.13) 250 \mathrm{GeV}=65 \mathrm{GeV}
$$

where $R$ now specifically labels the lowest-mass Higgs - i.e, the only one so far observed. This result suggests that the lowest-mass Higgson will effectively couple to the Higgs boson about as strongly as a neutralino with a mass of several hundred $\mathrm{GeV}$ and with an optimal admixture of Higgsino and neutral gaugino components.

There has recently been a trend toward pessimism regarding both supersymmetry and dark matter WIMPs $[8,9,10]$, but the tension between experiment/observation and theory is ameliorated if the neutralino is a less than dominant component of the dark matter [11]. There is then the difficulty of finding another well-motivated component which is also stable and fully consistent with observation. In the present scenario the Higgson perfectly fits this role because of both its similarities to the neutralino and its differences. It is made stable by an R-parity of -1 , it has similar weak and Higgs-mediated couplings and a mass near the electroweak scale (as required 
by most direct, indirect, and collider detection experiments), and it should therefore also share the "WIMP miracle" of producing about the right relic abundance, On the other hand, the mass is firmly predicted to be $\leq 125 \mathrm{GeV} / \mathrm{c}^{2}$, and there is no ambiguity like the arbitrariness of the parameters specifying the lowest-mass neutralino.

In the present scenario, the dominant dark matter component is thus much better characterized with respect to both mass and couplings than any other dark matter candidate.

The present scenario is readily falsified by experiment and observation. For example, if the positron excess observed by AMS [12] (and other experiments) had demonstrated that the dominant dark matter particle has a mass of roughly $800 \mathrm{GeV}$ or above, the rigorous limit of $125 \mathrm{GeV}$ in the present scenario would have been disconfirmed. But the Planck observations have instead disconfirmed this interpretation [13]. On the other hand, the interpretation of a low-mass darkmatter signal in the analyses cited below is quite consistent with the Planck data, and in addition is just as expected for thermal production.

Three other sets of observations are in agreement with our $125 \mathrm{GeV}$ limit: the gamma ray excess from the Galactic center [14, 15], the gamma ray excess from Omega Centauri [16] (interpreted as a stripped and nearby dwarf galaxy), and the antiprotons observed by AMS [17, 18]. The significance of these results is typically estimated to lie between about 3 and $5 \sigma$, depending on the models used, but these are three independent sets of data with analyses by several independent groups.

The constraints on the couplings and mass of the Higgson are consistent with the CMS and ATLAS constraints on the branching ratio for invisible Higgs decays [19, 20, 21].

The present candidate has one extremely novel feature: It requires a mild violation of Lorentz invariance in this new sector at high energy, with one component of the action not being invariant under a Lorentz boost (so that there is a preferred frame - the cosmological frame). Rotational invariance and gauge invariance are preserved in all sectors, and full Lorentz invariance is preserved as long as the internal degrees of freedom in $\Phi$ are not excited, so that only the Higgs/amplitude mode is observed. All of standard physics is preserved (with full Lorentz invariance), including grand unification and supersymmetry. (In fact, the fundamental theory behind the present scenario cannot even be formulated without both $\mathrm{SO}(10)$ grand unification and supersymmetry [22]. Here, however, we regard the Lagrangian of Refs. $[4,5,6,7]$ as being phenomenological rather than derived from Ref. [22].) The spin-statistics theorem does not apply to the Higgson sector, as it also does not in nonrelativistic physics [23], and the Higgsons are then allowed to have spin 1/2, as is required to preserve rotational invariance for one term in the Lagrangian.

It is interesting, however, that many of the familiar features of special relativity remain even in the Higgson sector, even though strict Lorentz invariance and the spin-statistics theorem are lost. For example, the Higgson kinetic action is

$$
S_{i}=\int d^{4} x H^{i \dagger}(x)\left(D^{\mu} D_{\mu}-M_{i}^{2} c^{2}\right) H^{i}(x)
$$

where we deviate from natural units by including factors of $c$ while keeping $\hbar=1$. (Summation over the indices labeling fields is never implied, but the summation convention is always used for coordinate indices like $\mu$.) This gives the usual relativistic equation of motion $\left(-D^{\mu} D_{\mu}+M_{i}^{2} c^{2}\right) H^{i}(x)=$ 0 . For a free particle in a momentum eigenstate $H^{i}(x)=H_{0}^{i} e^{i p_{\mu} x^{\mu}}$, this implies that $-p_{0}^{2}+p^{2}+$ 
$M_{i}^{2} c^{2}=0$, where $p^{2}=p^{k} p_{k}$ with $k=1,2,3$, or

$$
E^{2}=p^{2} c^{2}+M_{i}^{2} c^{4}
$$

since $E=p_{0} c$. Wave-particle duality implies that the particle velocity $v$ is the group velocity of the wave, $v=d E / d p$, and by definition the inertial mass $m$ is the ratio of the momentum to the velocity, $m=p / v$. Eq. (6) then implies $2 E d E / d p=2 p c^{2}$ or

$$
E=m c^{2}
$$

just as for more familiar particles.

There are, of course, many hazards to be considered with even a mild violation of Lorentz invariance in a single sector that is at the current edge of experimental accessibility, and a more detailed discussion is given in Ref. 7.

But again, all aspects of the theory have full Lorentz invariance until we reach this one sector at high energy. There are a number of examples of previously unimaginable violations of symmetries arising as new sectors of physics become accessible - principally P, CP, and T violation in 1956, 1964, and 2012 [24], with important consequences such as the distinction between left- and righthanded fields and the presence of a third generation of fermions. In the present case, there is a compelling need for a natural resolution of the difficulty of explaining dark matter with only a supersymmetric WIMP, while still preserving the successes of supersymmetry.

It should also not be surprising that the Higgs sector may be richer than originally envisioned, since exploration of this sector has only begun. (There were many surprising enrichments of physics following the introduction of the spin 1/2 and spin 1 sectors, in 1897 and 1905 respectively. J.J. Thomson could not have anticipated that the electron would be described by a four-component quantum field, and Einstein could not have anticipated the richness of quantum electrodynamics.) Finally, there is the inspiration and precedent of the Higgs modes in superconductors, which are also amplitude modes in a richer structure [25].

\section{References}

[1] H. Baer and X. Tata, Weak Scale Supersymmetry: From Superfields to Scattering Events, Cambridge University Press, Cambridge 2006, and references therein.

[2] Gordon Kane, editor, Perspectives on Supersymmetry II, World Scientific, Singapore 2010, and references therein.

[3] G. Jungman, M. Kamionkowski, and K. Griest, Supersymmetric Dark Matter, Phys. Reports 267, 195 (1996), arXiv:hep-ph/9506380, and references therein.

[4] Roland E. Allen and Aritra Saha, Dark matter candidate with well-defined mass and couplings, Mod. Phys. Lett. A 32, 1730022 (2017), arXiv:1706.00882 [hep-ph].

[5] Roland E. Allen, Saving supersymmetry and dark matter WIMPs - a new kind of dark matter candidate with well-defined mass and couplings, Phys. Scr. 94, 014010 (2019), arXiv:1811.00670 [hep-ph].

[6] Maxwell Throm, Reagan Thornberry, John Killough, Brian Sun, Gentill Abdulla, and Roland E. Allen, Two natural scenarios for dark matter particles coexisting with supersymmetry, Mod. Phys. Lett. A 34, 1930001 (2019), arXiv:1901.02781 [hep-ph]. 
[7] Reagan Thornberry, Maxwell Throm, John Killough, Dylan Blend, Michael Erickson, Brian Sun, Brett Bays, Gabe Frohaug, and Roland E. Allen, A natural multicomponent dark matter scenario with two coexisting stable WIMPs, submitted.

[8] Michael E. Peskin, Supersymmetric dark matter in the harsh light of the Large Hadron Collider, Proc. Natl. Acad. Sci. USA 112, 12256 (2015).

[9] Keith A. Olive, Supersymmetric Dark Matter or Not, proceedings of 11th International Workshop on Dark Side of the Universe, arXiv:1604.07336 [hep-ph].

[10] H. Baer, V. Barger, D. Mickelson, and M. Padeffke-Kirkland, SUSY models under siege: LHC constraints and electroweak fine-tuning, Phys. Rev. D89, 115019 (2014), arXiv:1404.2277 [hep-ph].

[11] Howard Baer, Vernon Barger, Dibyashree Sengupta, and Xerxes Tata, Is natural higgsino-only dark matter excluded?, arXiv:1803.11210 [hep-ph].

[12] Dan Hooper, Ilias Cholis, Tim Linden, and Ke Fang, HAWC Observations Strongly Favor Pulsar Interpretations of the Cosmic-Ray Positron Excess, Phys. Rev. D 96, 103013 (2017), arXiv:1702.08436 [astro-ph.HE], and references therein.

[13] Planck Collaboration, arXiv:1807.06209 [astro-ph.CO]. See Fig. 46 in particular.

[14] Christopher Karwin, Simona Murgia, Tim M. P. Tait, Troy A. Porter, and Philip Tanedo, Dark matter interpretation of the Fermi-LAT observation toward the Galactic Center, Phys. Rev. D 95, 103005 (2017), arXiv:1612.05687 [hep-ph], and references therein.

[15] Rebecca K. Leane and Tracy R. Slatyer, Dark Matter Strikes Back at the Galactic Center, arXiv:1904.08430 [astro-ph.HE], and references therein.

[16] Anthony M. Brown, Richard Massey, Thomas Lacroix, Louis E. Strigari, Azadeh Fattahi, and Céline Boehm, The glow of annihilating dark matter in Omega Centauri, arXiv:1907.08564 [astro-ph.HE].

[17] Ilias Cholis, Tim Linden, and Dan Hooper, A Robust Excess in the Cosmic-Ray Antiproton Spectrum: Implications for Annihilating Dark Matter, Phys. Rev. D 99, 103026 (2019), arXiv:1903.02549 [astro-ph.HE].

[18] Alessandro Cuoco, Jan Heisig, Lukas Klamt, Michael Korsmeier, and Michael Krämer, Scrutinizing the evidence for dark matter in cosmic-ray antiprotons, Phys. Rev. D 99, 103014 (2019), arXiv:1903.01472 [astro-ph.HE].

[19] CMS Collaboration, Search for invisible decays of a Higgs boson produced through vector boson fusion in proton-proton collisions at $\sqrt{s}=13 \mathrm{TeV}$, Phys. Lett. B 793, 520 (2019), arXiv:1809.05937 [hep-ex].

[20] M. Aaboud et al. (ATLAS Collaboration), Combination of Searches for Invisible Higgs Boson Decays with the ATLAS Experiment, Phys. Rev. Lett. 122, 231801 (2019), arXiv:1904.05105 [hep-ex].

[21] Giorgio Arcadi, Abdelhak Djouadi, and Martti Raidal, Dark Matter through the Higgs portal, arXiv:1903.03616 [hep-ph], and references therein.

[22] R. E. Allen, Predictions of a fundamental statistical picture, arXiv:1101.0586 [hep-th].

[23] R. E. Allen and A. R. Mondragon, No spin-statistics connection in nonrelativistic quantum mechanics, arXiv:quant-ph/0304088, Phys. Rev. A 68, 046101 (2003).

[24] J. P. Lees et al. (The BABAR Collaboration), Observation of Time-Reversal Violation in the $B^{0}$ Meson System Phys. Rev. Lett. 109, 211801 (2012), arXiv:1207.5832 [hep-ex].

[25] D. Pekker and C. M. Varma, Amplitude/Higgs Modes in Condensed Matter Physics, Annu. Rev. Condens. Matter Phys. 6, 269 (2015). 